\title{
A Rare Case of Pulmonary Arteriovenous Hemangioma Presenting as a Peribronchial Mass
}

\author{
Soomin Ahn · Sejin Jung · Jong Ho Cho ${ }^{1}$ Tae Sung $\mathrm{Kim}^{2} \cdot$ Joungho Han \\ Departments of Pathology and Translational Genomics, ${ }^{\top}$ Thoracic Surgery, and ${ }^{2}$ Radiology, Samsung Medical Center, \\ Sungkyunkwan University School of Medicine, Seoul, Korea
}

Vascular tumors in the lung are unusual. The majority of previous reports described cases of multiple or solitary lymphangiomas or capillary and cavernous hemangiomas. ${ }^{1-4}$ To date, there has been only one report describing arteriovenous malformation/hemangioma (AVMH) in the middle mediastinum. ${ }^{5}$ Herein, we report an unusual case of pulmonary AVMH presenting as a peribronchial mass.

\section{CASE REPORT}

A 62-year-old man presented with a 1-month history of sputum and fatigue. He was afebrile, and routine physical examinations were within normal limits. He is an ex-smoker with a 40 pack years smoking history and denied any other medical history. Routine laboratory tests showed no abnormality. Chest computed tomography (CT) identified a 45-mm-sized mass-like lesion located centrally in the apical segment of the left upper lobe (Fig. 1A). Although the CT findings were not diagnostic, the possibility of infectious condition was favored rather than neoplastic. The patient took antibiotics for 45 days, but experienced no symptom relief, and follow-up chest CT showed no interval change of the lesion. Additional positron emission tomography was performed to characterize the lesion. A $55 \times 24$-mm-sized soft tissue mass showed mild heterogeneous fludeoxyglucose up-

\section{Corresponding Author}

Joungho Han, MD

Department of Pathology and Translational Genomics, Samsung Medical Center,

Sungkyunkwan University School of Medicine, 81 Irwon-ro, Gangnam-gu, Seoul 06351, Korea

Tel: +82-2-3410-2765, Fax: +82-2-3410-0025, E-mail: hanjho@skku.edu

Received: July 29, 2015 Revised: September 16, 2015 Accepted: October 15, 2015 take in the lesion, suggesting malignancy (Fig. 1A). Under the clinical impression of lung cancer, left upper lobectomy was planned. Although video-assisted thoracoscopic surgery was initially attempted during the operation, it was converted to thoracotomy due to mediastinal pleural adhesion.

On the cut section of the lobectomy specimen, the dilated lumen of the lobar bronchus was packed with necrotic and mucoid material (Fig. 1B). Bronchiectasis was noted along the bronchial tree. There was no definite endobronchial lesion. Interestingly, serial cut sections of the peribronchial area revealed an ill-defined white-yellow rubbery mass-like lesion that measured approximately $2.8 \mathrm{~cm}$ in total extent and had pinpoint-sized spaces (Fig. 1B). Microscopically, the lesion consisted of multiple thick and prominent vessels with intervening connective tissue, and it grew between bronchial mucosa and hyaline cartilage plates (Fig. 1C). The vessels were variably sized, up to $4 \mathrm{~mm}$ in diameter with a thickness of up to $0.8 \mathrm{~mm}$ (Fig. 1D). Elastic stain visualized no definite elastic lamina, which implied the lesion to be composed of arterialized vein (Fig. 1E). These histologic findings were characteristic of AVMH. The patient was discharged without any postoperative complication. This study was approved by the Institutional Review Board of Samsung Medical Center (IRB File No. SMC 2015-07-194).

\section{DISCUSSION}

Vascular tumors of the lung are extremely rare. We presented an unusual case of pulmonary AVMH clinically mimicking lung cancer. This hemangioma presented as a peribronchial mass leading to post-obstructive bronchiectasis with mucin impaction.

The recent World Health Organization classification intro- 


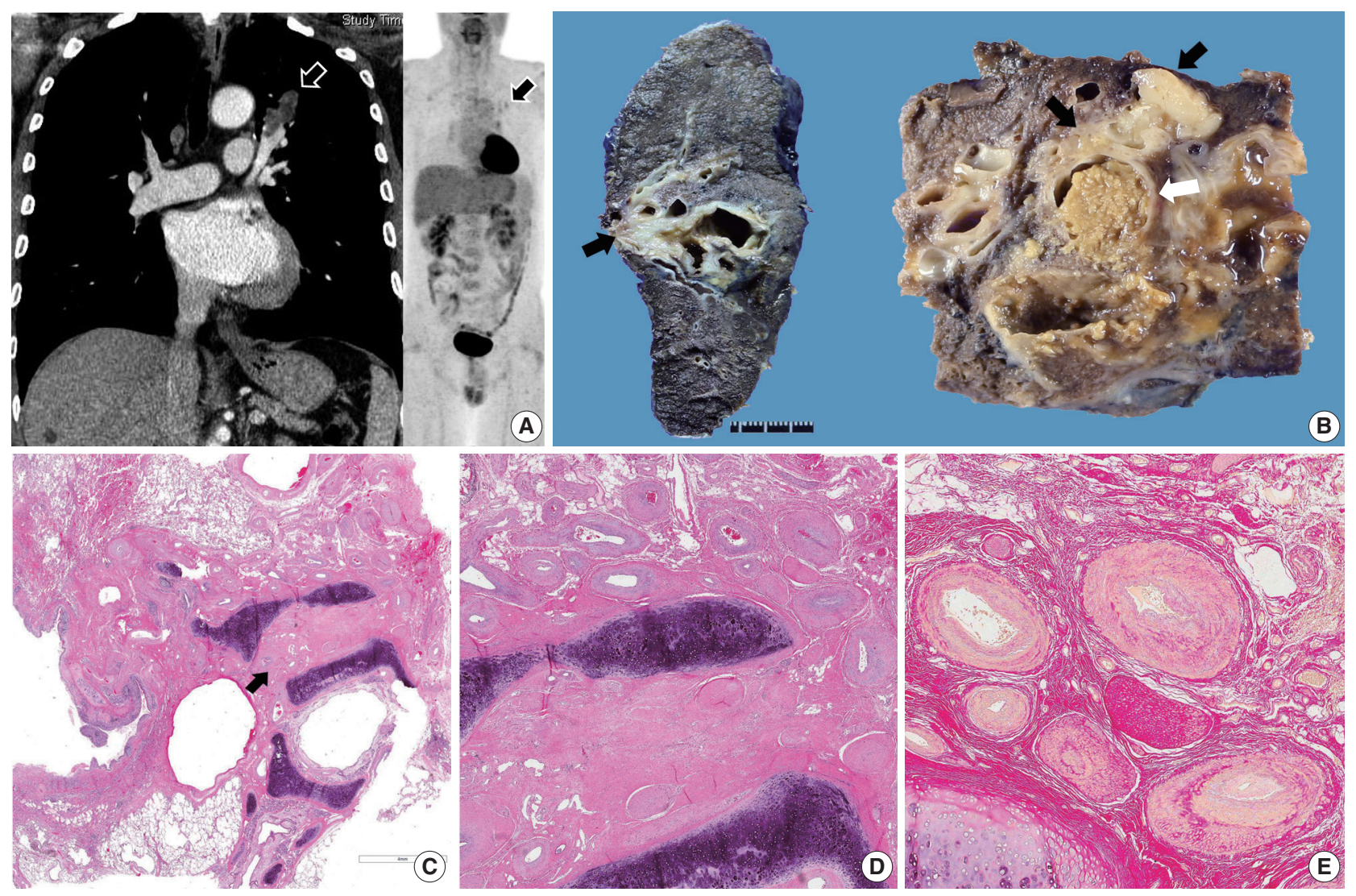

Fig. 1. Arteriovenous malformation/hemangioma (AVMH) of the lung. (A) Chest computed tomography reveals a mass-like lesion measuring $45 \mathrm{~mm}$, and the lesion shows mild heterogeneous fludeoxyglucose uptake on positron emission tomography scan. (B) On the cut section of the lobectomy specimen, an ill-defined rubbery mass-like lesion (black arrows) is noted adjacent to dilated bronchial lumen with mucin impaction (white arrow). Microscopically, the lesion grows between the bronchial mucosa and cartilage plate (C) and consists of multiple thick and prominent vessels of various sizes, consistent with the diagnosis of $\mathrm{AVMH}$ (D). (E) Elastic stain visualizes no definite elastic lamina.

duces four entities of vascular neoplasm in the mediastinum: lymphangioma, hemangioma, epithelioid hemangioendothelio$\mathrm{ma}$, and angiosarcoma. ${ }^{6}$ Of the benign entities, lymphangioma is composed of medium- or small-sized lymphatic channels filled with lymphatic fluid, and hemangioma can be cavernous or capillary. To date, there have been only a few reports of multiple or solitary lymphangiomas and hemangiomas in the lung. ${ }^{1-4}$ The present tumor had histologic features of AVMH characterized by complex thick-walled vessels. To the best of our knowledge, this is the first report describing AVMH arising in the peribronchial soft tissue. Deep-seated AVMH usually arises in the limbs or head and neck. Mizutani et al. previously reported arteriovenous hemangioma in the middle mediastinum.

The literature review showed that abnormal communications between pulmonary arteries and pulmonary veins have been given various names including pulmonary arteriovenous fistulae, arteriovenous aneurysm, and AVMH. ${ }^{7,8}$ Among these, pulmonary arteriovenous fistulae refer to abnormally dilated vessels that provide a right-to-left shunt between the pulmonary artery and pulmonary vein, thereby bypassing the pulmonary capillary bed. ${ }^{8}$ Pulmonary arteriovenous fistulae are mostly congenital, and the majority of patients have hereditary hemorrhagic telangiectasia. ${ }^{8}$ These lesions have been described as pulmonary AVMH in several reports. ${ }^{78}$ On the other hand, AVMH is different from arteriovenous fistulae in that AVMH is an acquired tumor-like condition characterized by complex thick-walled vessels with no evidence of fistula formation. In the present case, there was no evidence of obvious fistula or shunt formation on chest CT. To avoid misunderstanding and confusion, clarification of terminology for pulmonary vascular lesion is warranted.

\section{Conflicts of Interest}

No potential conflict of interest relevant to this article was reported. 


\section{REFERENCES}

1. Fugo K, Matsuno Y, Okamoto K, et al. Solitary capillary hemangioma of the lung: report of 2 resected cases detected by high-resolution CT. Am J Surg Pathol 2006; 30: 750-3.

2. Song HJ, Han J, Kim K, Lee KS, Seo J. Solitary pulmonary lymphangioma in an adult: a brief case report. Korean J Pathol 2008; 42: 125-7.

3. Maeda R, Isowa N, Sumitomo S, Matsuoka K. Pulmonary cavernous hemangioma. Gen Thorac Cardiovasc Surg 2007; 55: 177-9.

4. Weissferdt A, Moran CA. Primary vascular tumors of the lungs: a review. Ann Diagn Pathol 2010; 14: 296-308.

5. Mizutani E, Morita R, Kitamura S. Arteriovenous hemangioma in the middle mediastinum: report of a case. Surg Today 2011; 41: 846-8.

6. Travis WD, Brambilla E, Burke AP, Marx A, Nicholson AG. WHO classification of tumours of the lung, pleura, thymus and heart. Lyon: IARC Press, 2015.

7. Gossage JR, Kanj G. Pulmonary arteriovenous malformations: a state of the art review. Am J Respir Crit Care Med 1998; 158: 643-61.

8. Gill SS, Roddie ME, Shovlin CL, Jackson JE. Pulmonary arteriovenous malformations and their mimics. Clin Radiol 2015; 70: 96-110. 